\title{
Modernas instalaciones con molinos tubulares
}

PETER TICGESBAEUMKER

Polysius, S. A.

\section{N T R O D U C C I O N}

Examinando las nuevas plantas instaladas durante los últimos años en las fábricas de cemento, se observan dos características esenciales:

En primer lugar, llama la atención el incremento constante de la capacidad de producción individual de los molinos (fig. 1). En segundo lugar, se reconoce que existe un interés creciente en los sistemas económicos, en cuanto al consumo de energía.

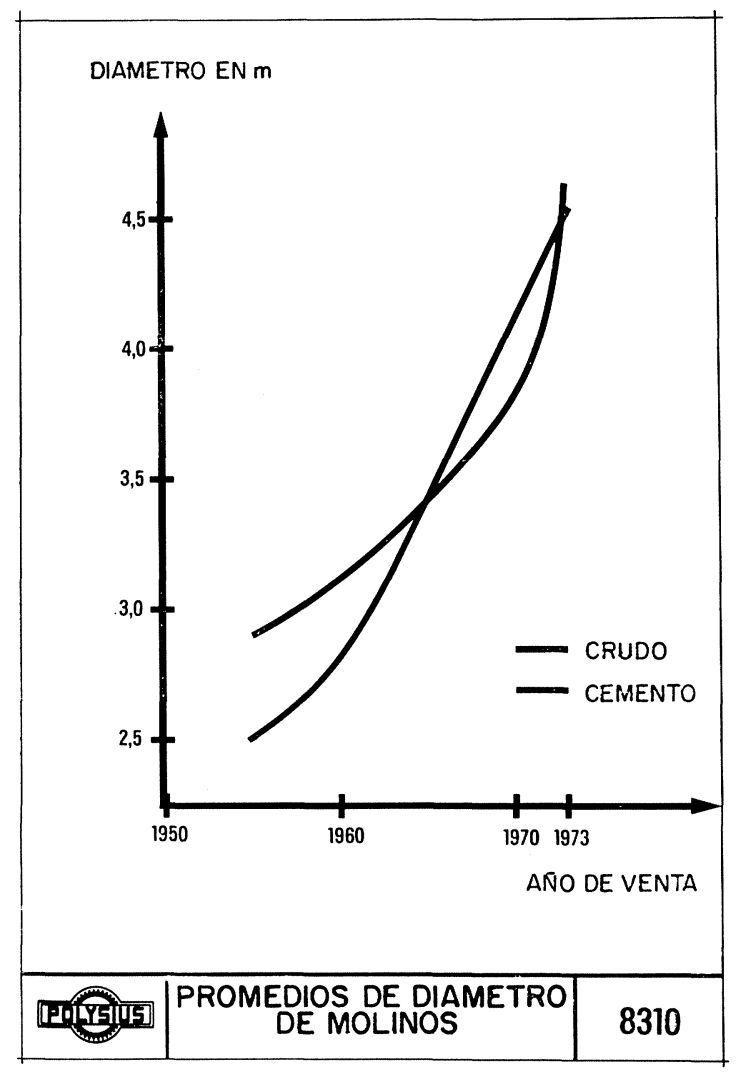

Fig. 1.-Promedio de diámetros de molinos de bolas.

Los molinos siguen, con ello, al desarrollo de los hornos hacia unidades cada vez más grandes. El motivo principal para esta tendencia hemos de buscarlo, sin duda, en los costos de fabricación. Evidentemente, parece claro, a primera vista, que los costos relativos de inversión, personal $\mathrm{y}$, al menos en el caso de los hornos - también de energía-, disminuyen cuanto más crecen los tamaños de las unidades de producción. En los molinos grandes no se obtienen, en general, ahorros apreciables de energía eléctrica, como tampoco desde el punto de vista del desgaste específico se presentan ventajas en comparación con unidades pequeñas.

La necesidad del ahorro de energía no precisa de comentarios: lo podemos leer a diario en la prensa. No en balde son necesarios para la fabricación de una tonelada de cemento unos $100 \mathrm{kWh}$; esto quiere decir que el consumo de energía eléctrica de una fábrica con una producción de 4.000 t/día de cemento es el mismo de una ciudad de 35.000 habitantes. Si sumamos el consumo total de energía por todos los conceptos, y lo expresamos en kcal, llegamos a la cifra, nada despreciable, de $1.000 .000 \mathrm{kcal} / \mathrm{t}$ de cemento.

En un horno moderno con intercambiador de calor ya se alcanza al menos un grado de rendimiento de un $56 \%$, y este rendimiento puede todavía ser mejorado hasta un $66 \%$ al aprovechar el calor sobrante para el secado del crudo. 


\section{INSTALACION PARA LA MOLIENDA DE CRUDOS}

Lo antes expuesto deja claro que debe prestarse la máxima atención a la elección del sistema de molturación del crudo. Ante todo, el consumo de energía eléctrica para la molienda debe ser bajo, y para el secado debe aprovecharse el calor sobrante del horno para precisar un mínimo de calor adicional. Aparte, deben considerarse otros puntos de vista en la elección del sistema de molienda más idóneo, como son:

- los costos de inversión;

- la necesidad de espacio;

- la simplicidad de operación;

- el acopio y almacenamiento de repuestos;

- los costos de mantenimiento;

- la disponibilidad y, naturalmente,

- el crudo a moler.

De este último son fundamentales su tamaño de alimentación, su humedad, su deslizamiento, su comportamiento en la fragmentación, su molturabilidad y su abrasividad; condicionantes, todos ellos, que determinan la elección del sistema de molienda.

En lo que sigue, se resumirán brevemente los distintos sistemas modernos de molienda y secado mediante molinos tubulares.

Si bien el molino vertical constituye, en muchos casos, una solución interesante, en esta ponencia no nos referiremos a él, ya que en otro momento de estos Coloquios se informará extensamente sobre este tema.

Puesto que el proceso de vía húmeda pierde de año en año importancia en la industria cementera, tampoco se va a hablar aquí sobre los molinos de pasta.

\section{Molino barrido por aire}

El primer tipo de molino empleado para instalaciones de molienda y secado fue el barrido por aire, del que la figura 2 contiene una sección. La característica de este molino es que trabaja en circuito cerrado con un separador estático hacia donde el material es llevado, arrastrado por una corriente de gas. Este transporte neumático requiere un elevado caudal de gas, por lo que este sistema presenta una buena posibilidad de secar en el molino. Admite una humedad del crudo de hasta un $15 \%$, y, caso de usar exclusivamente los gases calientes del horno correspondiente, se puede secar hasta el $8 \%$ de humedad inicial sin aportación de calor adicional. Ventaja esencial del molino barrido por aire es la sencilla disposición de los distintos equipos y de toda la instalación, lo que, unido a la excelente aptitud para el secado, facilita la operación y el mantenimiento no presenta problemas. La regulación se resuelve fácilmente a través del grado de llenado del molino. El mayor inconveniente estriba en el elevado consumo de energía del transporte neumático, que se debe cifrar en 2 a $3 \mathrm{kWh} / \mathrm{t}$ de producto terminado.

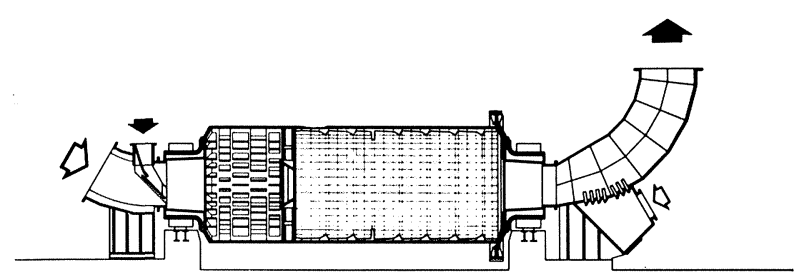

Fig. 2.-Sección de un molino barrido por aire.

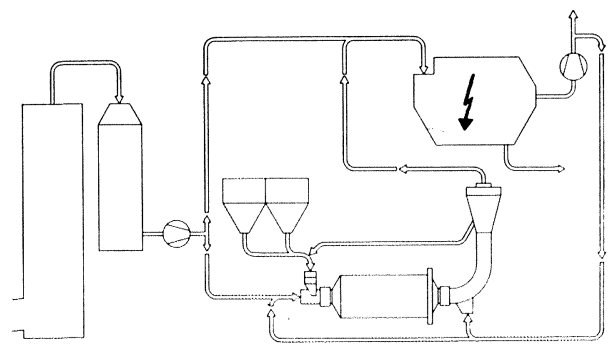

Fig. 3.-Esquema de una instalación con molino barrido por aire. 
La figura 3 representa el esquema de flujos de una instalación con aprovechamiento del calor sobrante de un horno con intercambiador. La disposición con el exhaustor del horno detrás de la torre de refrigeración es la misma que se conoce de instalaciones con molino vertical.

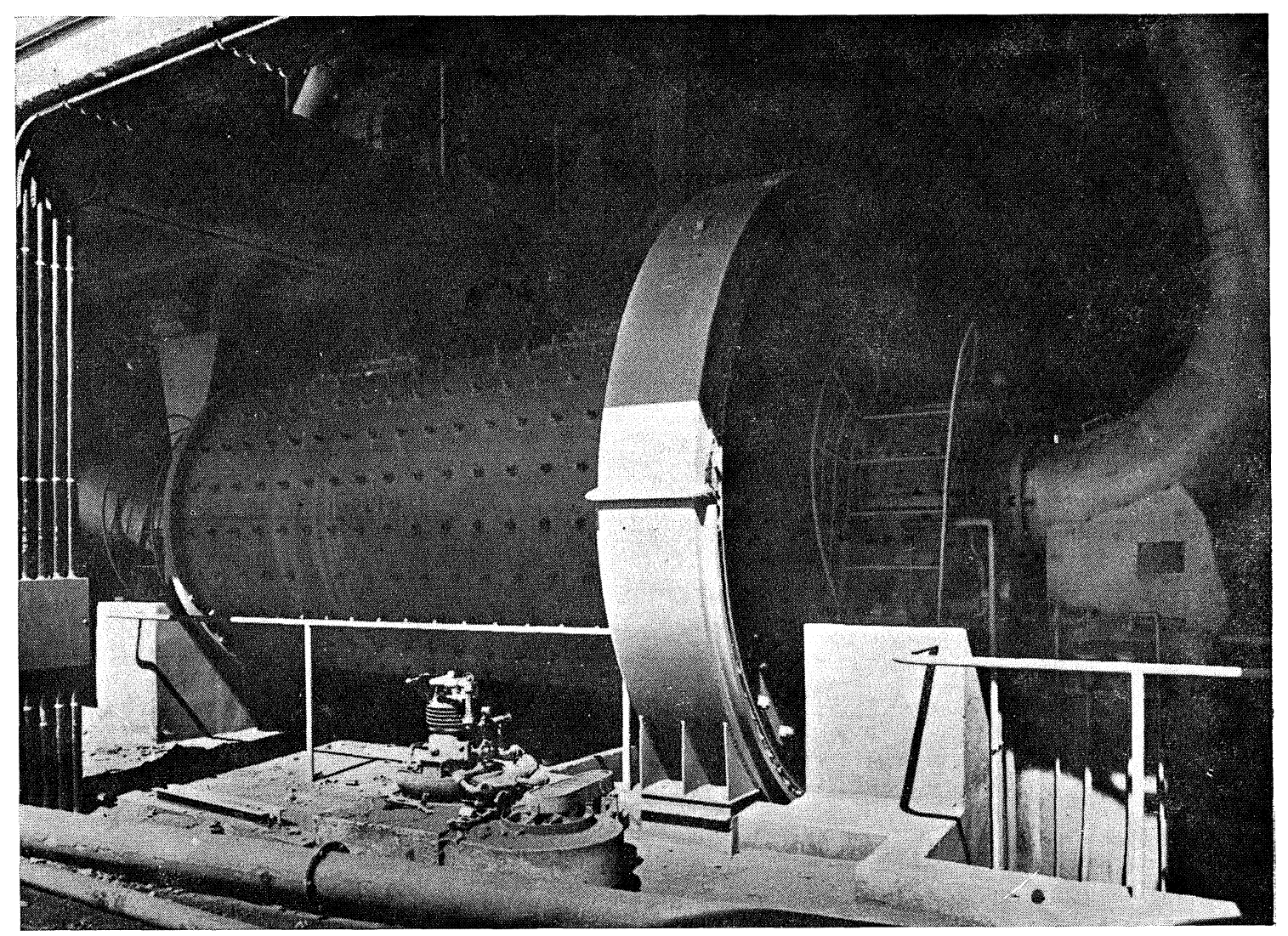

Fig. 4.-Fotografía de una instalación con molino barrido por aire.

En la figura 4 puede verse una fotografía de un molino barrido por aire.

\section{Molinos de descarga final y circuito por elevador}

El transporte neumático en el caso del molino barrido por aire es sustituido por un transporte con medios mecánicos, con el fin de ahorrar energía. Este tipo de molino lo llamamos molino de descarga final.

La figura 5 representa una sección. Contrariamente al molino barrido por aire antes descrito, aquí la descarga del material molido al final del molino se efectúa mecánicamente a través de una pared elevadora.

En este molino, normalmente de una sola cámara de molienda, se mantiene una velocidad de paso de gas relativamente baja para favorecer la molienda, quedando limitada, sin embargo, la posibilidad de secado. El máximo de humedad admisible es del $8 \%$, y sólo el $4 \%$ en caso de utilizar gases de escape del horno exclusivamente.

La granulometría del material alimentado no debe sobrepasar esencialmente los $15 \mathrm{~mm}$, con el fin de poder ajustar óptimamente la carga de bolas para la pre-molienda y el refino en una única cámara.

La figura 6 muestra un esquema de flujos: el molino en circuito cerrado con separador centrífugo, un separador estático intercalado con el conducto de gas de escape sirve 
para descargar aquél, ya que permite ajustar la finura del polvo decantado en el filtro a la finura del producto terminado.

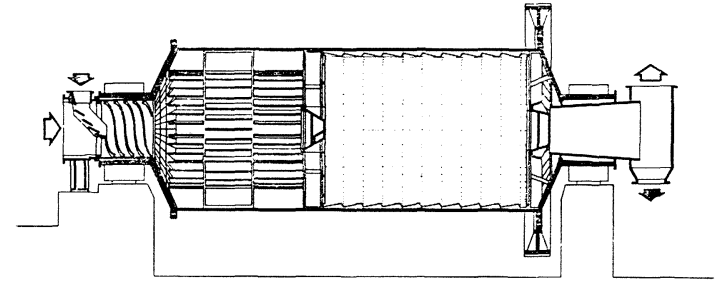

Fig. 5.-Sección de un molino con descarga en un extremo.

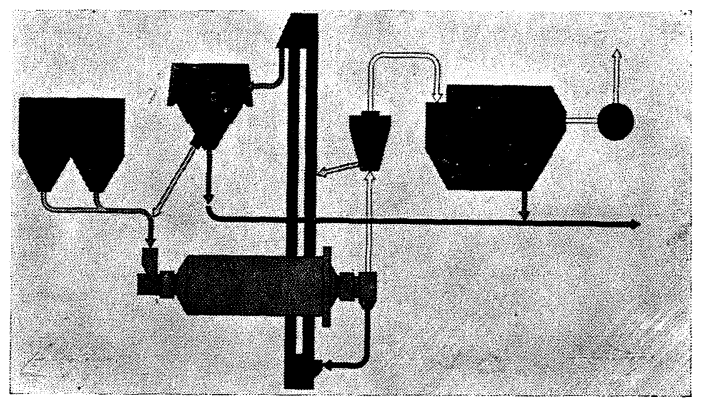

Fig. 6.-Esquema de un molino con descarga en un extremo.

La ventaja más significativa de este tipo de molino, en comparación con el molino barrido por aire, es el consumo inferior de energía. También es fácil efectuar una regulación en dependencia de la carga circulante.

Para altas producciones es posible reunir dos molinos, de una cámara cada uno, en un solo tubo de molino. La alimentación se realiza, entonces, a través de los dos extremos, y el material molido sale por orificios en la mitad del tubo para ser llevado independientemente a dos separadores centrífugos. Se trata, por tanto, de dos circuitos de molienda separados; en definitiva, dos molinos unidos en un solo cilindro.

\section{Doble-Rotador}

Reuniendo las ventajas del molino barrido por aire, esto es, gran caudal de gas y con ello buena posibilidad de secado, con las ventajas del molino de descarga final, que son: bajo consumo de energía por molienda óptima y circuito cerrado, se llega al Doble-Rotador, del cual la figura 7 muestra una sección.

La original disposición de las cámaras de molienda con la alimentación por los dos extremos y la descarga conjunta por el centro permite equipar las cámaras conforme al trabajo que han de realizar, tanto en cuanto a la conformación del blindaje como a la composición de la carga de bolas y, al mismo tiempo, adaptar la ventilación en la cámara de refino a las necesidades de la molienda, al realizar el secado casi exclusivamente en la parte anterior del molino. El caudal de gas necesario para el secado origina en el recinto de la pre-molienda una corriente de alta velocidad, pero aquí no es perjudicial, sino que, más bien, fomenta al flujo del material.

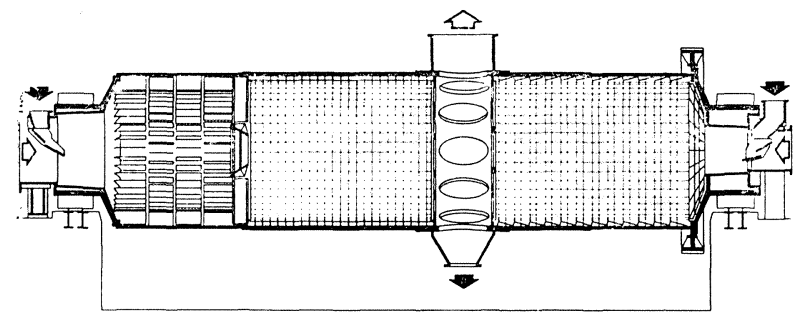

Fig. 7.-Sección de un Doble-Rotador.

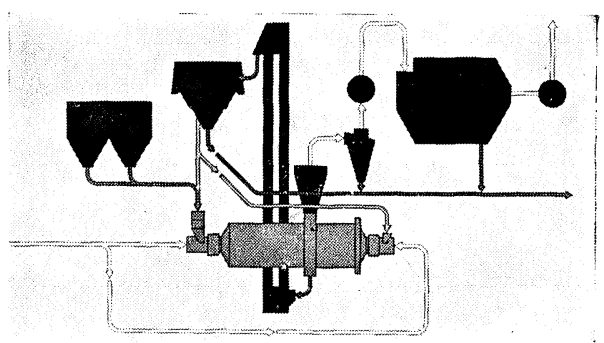

Fig. 8.-Esquema de un Doble-Rotador.

La posibilidad de optimación antes apuntada significa un consumo de energía comparativamente bajo, pudiendo ser el grosor del material alimentado bastante mayor que en los tipos de molino antes contemplados. 
La figura 8 es un esquema de flujos de una instalación con Doble-Rotador. El material procedente de las dos cámaras es llevado conjuntamente al separador centrífugo. Los gruesos son conducidos a la cámara de refino en su mayor parte; sólo una pequeña parte se reintroduce en la primera cámara, más que nada para empolvar el material húmedo y, con ello, facilitar su deslizamiento.

Para la ventilación de la segunda cámara se desvía una pequeña parte del gas caliente, que se une otra vez a la corriente principal en la descarga en el centro, para luego ser llevadas conjuntamente a la instalación de captación de polvo. También aquí existe un separador estático en el conducto del gas que permite adecuar la finura del polvo a la del producto terminado.

Las figuras 9 y 9.1 muestran un molino Doble-Rotador.

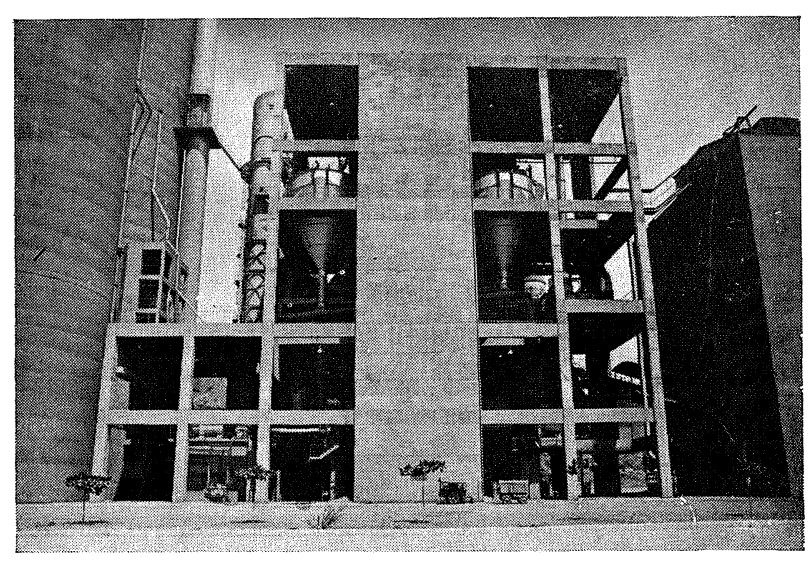

Fig. 9.-Fotografía de una instalación con Doble-Rotador.

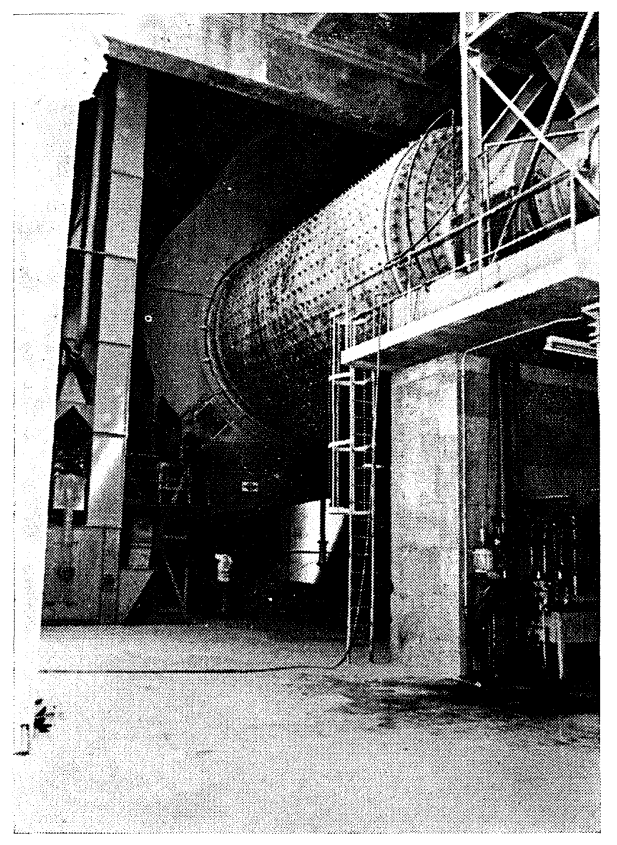

Fig. 9.1

Como consecuencia de la posibilidad de pasar grandes cantidades de aire, podemos secar en este molino humedades de hasta un $14 \%$, o bien un $7 \%$, en el caso de disponer exclusivamente de gases de escape de un horno con intercambiador de ciclones.

\section{Instalaciones de molienda con machacadoras y secadores previos}

Si por el proceso de molturación es conveniente el emplear una de las clases de molinos

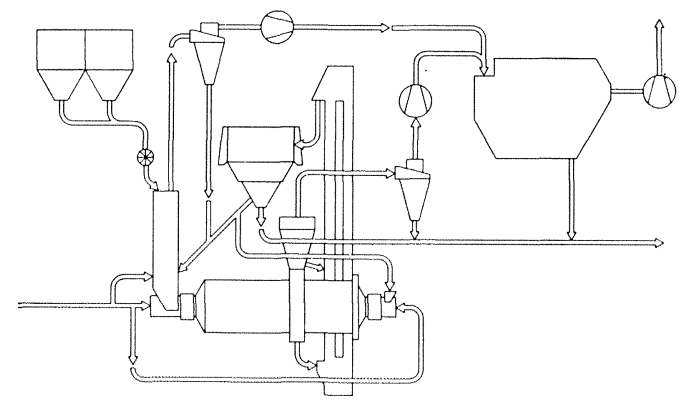

Fig. 10.-Esquema de una instalación de molienda con secador. anteriormente descritas, pero, sin embargo, el material a alimentar tiene una humedad muy alta y un tamaño muy grande, pueden instalarse previamente diversos aparatos para acondicionar el material convenientemente.

\section{Secadores verticales}

En la figura 10 puede verse el esquema de flujos de una instalación con Doble-Rotador y un secadero vertical previo. El material 
pasa este secadero en contracorriente con los gases calientes, llegando finalmente al molino con una humedad residual apta para el mismo. En la parte inferior del secadero puede estar instalada también una machacadora. Instalaciones de este tipo pueden trabajar con materiales de hasta el $20 \%$ de humedad inicial.

\section{Secadores de tambor}

Estos secadores se emplean para materiales de difícil secado. Con ellos se puede llegar a evaporar hasta un $25 \%$ de humedad. Se distingue principalmente entre dos tipos: el secadero de tambor largo y de pequeña velocidad y el secadero rápido de tambor corto.

En fábricas de cemento hay instalados secadores lentos con una capacidad de evaporación de hasta $40.000 \mathrm{~kg} / \mathrm{h}$ de agua. Con este tipo de secador se pueden manipular materiales de características muy difíciles.

Secadores rotativos rápidos están funcionando en fábricas de cemento hasta capacidades de unos $20.000 \mathrm{~kg} / \mathrm{h}$ de agua evaporada. Este tipo de secadero es prácticamente una cámara de secado independiente del molino. El rendimiento referido al volumen de tambor que se consigue en ellos es, aproximadamente, el doble de los secadores lentos.

\section{Machacadoras con calefacción}

En el caso de que no solamente haya de secarse el material antes de su entrada al molino, sino también sea necesaria una reducción de su tamaño, se emplean machacadoras con calefacción, por ejemplo: machacadora-secadora de impacto.

La figura 11 muestra una instalación de un molino, en este caso barrido por aire, con una machacadora-secadora previa. En este tipo de máquina se pueden preparar, para poder ser alimentados al molino, materiales con una humedad inicial de hasta el $25 \%$ y con un tamaño de hasta unos $200 \mathrm{~mm}$. El material tratado en la machacadora pasa directamente al molino.

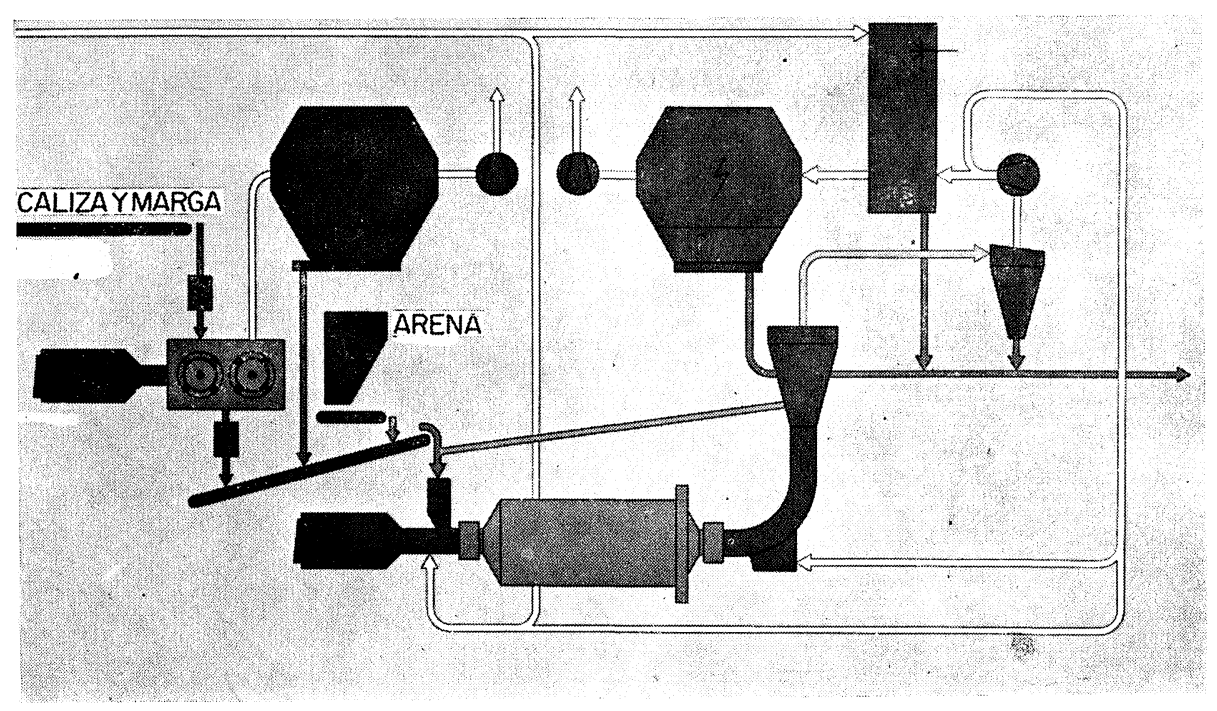

Fig. 11.-Esquema de una instalación de molienda con trituradora-secadora.

Los finos producidos por la machacadora pueden añadirse al producto final, si se toman las medidas adecuadas de clasificación previa. 
En casos específicos es posible alimentar el total del material machacado al turboseparador de la instalación del molino. En el turboseparador hay que secar entonces hasta la humedad residual final. No obstante, esta solución encierra ciertos inconvenientes para el separador, por lo que normalmente se debe prever una alimentación directa de la machacardora al molino.

Un inconveniente típico de las instalaciones de molienda con machacadora previa, es la pérdida de producción paulatina del molino al ir desgastándose los martillos de la machacadora y aumentando, por lo tanto, la granulometría de entrada en el molino. La instalación de machacadoras previas a molino no es, pues, conveniente si el material de alimentación es muy abrasivo.

\section{Molinos autógenos}

A pesar de que este tipo de molino no tiene mucha importancia en la industria cementera, se menciona para completar la relación.

Es un molino de gran diámetro y poca longitud, y se le llama autógeno porque aprovecha la energía de caída, del propio material para su molturación. No obstante, para la molienda de materia cruda para cemento hace falta una, aunque muy pequeña, carga de bolas.

Se le emplea para moler materiales difíciles, con mucha humedad e inclusiones de trozos de gran tamaño. Un inconveniente es su gran costo de inversión, ya que de todas formas se necesita instalar otro molino de refino.

En este lugar debería hablarse de molinos verticales de rodillos, pero, como se ha dicho anteriormente, son tema de otra ponencia.

En la tabla 1 se dan datos de instalaciones con algunos de los sistemas antes relatados.

TAB L A 1

Instalaciones modernas para molienda y secado de materia cruda

\begin{tabular}{|c|c|c|c|c|c|c|c|c|c|c|}
\hline Planta & $\begin{array}{l}\text { Dimensiones } \\
\mathbf{m} \varnothing \times \mathbf{m} \text { lgo. }\end{array}$ & Tipo de molino & $\begin{array}{l}\text { Acciona- } \\
\text { miento } \\
k W\end{array}$ & $\begin{array}{c}\text { Tipo de } \\
\text { accionamiento }\end{array}$ & $\begin{array}{c}\text { Humedad } \\
\%\end{array}$ & & nura & $\% \mathbf{R}$ & $\begin{array}{l}\text { Produc- } \\
\text { ción } \\
t / h\end{array}$ & Observaciones \\
\hline 1 & $5,8 \times 14,75$ & Barrido por aire & 5.200 & 2 Piñones (s) & 12 & & $5 \%$ & R 0,2 & 320 & \\
\hline 2 & $5,0 \times 8,5$ & Barrido por aire & 2.900 & 2 Piñones & 26 & 10 & $\%$ & R 0,09 & 240 & $\begin{array}{l}\text { Trituradora-secadora } \\
\text { ante molino }\end{array}$ \\
\hline 3 & $4,8 \times 10,0$ & Descarga en el extremo & 3.400 & 2 Piñones & 4 & 3 & $\%$ & R 0,09 & 135 & \\
\hline 4 & $4,2 \times 9,75$ & Descarga en el extremo & 2.400 & Central & 11,6 & 1 & $\%$ & $\mathrm{R} \quad 0,2$ & 210 & Secadero dispersor \\
\hline 5 & $5,0 \times 18,0$ & Doblerrotador & 5.000 & 2 Piñones (s) & 5,5 & 1 & $\%$ & $\mathrm{R} \quad 0,2$ & 317 & \\
\hline 6 & $4,6 \times 15,25$ & Dolblerrotiadior & 3.400 & Central & 9,0 & 18 & $\%$ & R 0,09 & 240 & Secadero vertic al \\
\hline 7 & $4,6 \times 14,25$ & Doblerrotador & 3.900 & Motor anular & 4,5 & 20 & $\%$ & R 0,09 & 184 & \\
\hline 8 & $46 / 23$ & Molino vertical & 1.750 & Espocial & 10,0 & 12 & $\%$ & $\mathrm{R} 0,09$ & 220 & \\
\hline 9 & $46 / 26$ & Molino vertical & 2.100 & Especial & 7,0 & 12 & $\%$ & R 0,09 & 400 & \\
\hline
\end{tabular}

En la instalación 1 se necesita para el secado el gas caliente del intercambiador del horno y, además, el de un hogar auxiliar. En la instalación 2 se alimenta un material con $150 \mathrm{~mm}$ de tamaño máximo y una humedad del $26 \%$ en una machacadora-secadora de impactos, la cual lo pone en unas condiciones de tamaño y humedad apto para que pueda ser molido y terminado de secar con sólo el aprovechamiento de los gases de un horno con precalcinación. 
En la instalación 3 se alimenta material con un tamaño de hasta $15 \mathrm{~mm}$ y se muele hasta una finura del $3 \%$ de residuo en tamiz de $0,09 \mathrm{~mm}$.

La instalación 4 dispone de dos secaderos rotativos en paralelo. En el primero se seca con gas del horno y en el otro con aire de escape del enfriador. Estos dos secaderos sirven para acondicionar el gas cuando el molino de crudo está parado. El Doble-Rotador de la instalación 5 tiene una corona con dentado inclinado y es accionado a través de dos piñones.

El Doble-Rotador de la instalación 6 tiene accionamiento central. El dimensionamiento de este molino se realizó de tal manera que pueda secarse en él, usando la cantidad mínima de gases calientes, la humedad normal del material. En caso de aumento de esta humedad, se preseca el material en un secadero vertical previo. El Doble-Rotador de la planta 7 es accionado mediante motor anular.

Las instalaciones 8 y 9 con molinos verticales de rodillos completan la lista.

\section{INSTALACIONES PARA MOLIENDA DE CLINKER}

En los grandes molinos de clínker que se instalan en la actualidad es de gran importancia que el cemento producido corresponda a la constante demanda de mejor calidad. La instalación de molienda debe ofrecer una gran disponibilidad de marcha $\mathrm{y}$, además, debe poder moler a distintas finuras, cuando sólo se dispone de un molino para la producción total de la fábrica.

Además de tener que producir una curva granulométrica adecuada para obtener unas buenas resistencias, existe la necesidad de que la temperatura durante la molienda no suba en exceso y que el cemento terminado esté lo más frío posible. En caso contrario, pueden aparecer dificultades de fraguado falso o rápido.

\section{Molino Compound (Circuito abierto)}

Partiendo de las instalaciones con molino previo y de refino, se desarrolló el molino Compound. La premolienda y el refino se hacen en un solo molino. El producto de salida del molino es un producto terminado. Actualmente se emplean principalmente molinos de dos cámaras, dotada la última de blindaje clasificador de bolas.

Es de mucha importancia para estos molinos tanto una correcta composición de la carga de bolas, como la relación de longitudes de las cámaras de molienda y el ajuste adecuado de la velocidad de paso del material de una cámara a la otra. El paso del material se puede influenciar variando la superficie libre de las placas ranuradas y también mediante la forma, el número y la longitud de las palas elevadoras. Hoy en día existen paredes elevadoras con la posibilidad de variar rápidamente, e incluso de manera continua, la cantidad del material transportado, mediante variación de la posición de las palas.

La figura 12 es una fotografía de un molino Compound. Los molinos Compound están preparados para trabajar normalmente hasta una finura de $3.200 \mathrm{~cm}^{2} / \mathrm{g}$ según Blaine. Hasta aquí trabajan con una rentabilidad igual que los molinos de dos cámaras con separador, de los cuales posteriormente hablaremos.

La desventaja principal de los molinos Compound radica en la dificultad del cambio rápido a otras finuras. El cambio definura solamente es posible con el cambio de la carga de bolas. En límites muy estrechos se puede también variar la finura, modificando la cantidad del material de alimentación. 
Otra de las dificultades es la disipación del calor latente del clínker y de molturación. Una parte de este calor es disipada por la radiación del molino y por convención, pero

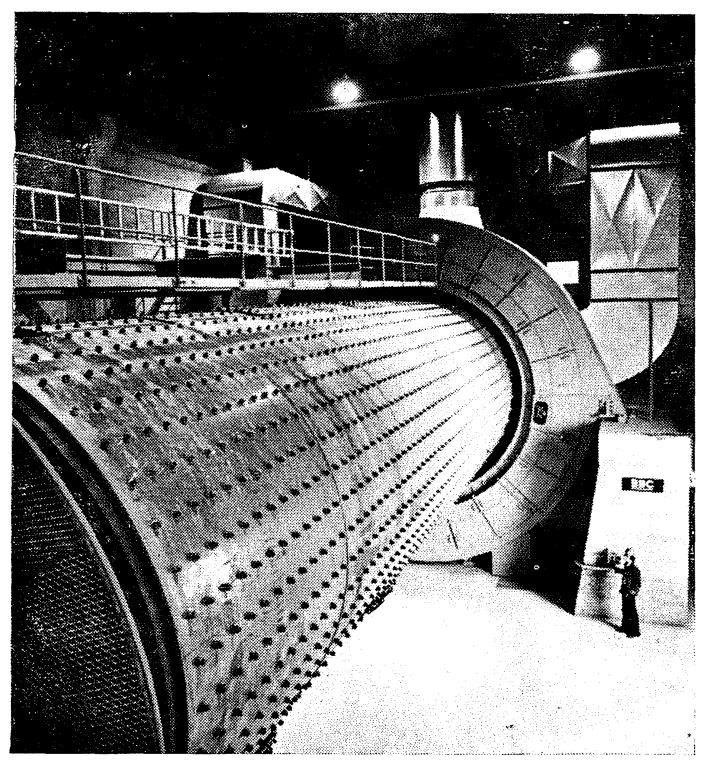

Fig. 12.-Foto de un molino "Compound". la mayor parte se elimina a través del aire de ventilación del molino. La ventilación del molino de cemento tiene, sin embargo, su límite, puesto que el arrastre de material puede llegar a perturbar la molienda. En los grandes molinos, este límite es más bajo que en los pequeños, es decir, cuanto mayor es el diámetro del molino, tanto menor es el caudal de aire por tonelada de cemento admisible. Esto tiene su explicación en el hecho de que la potencia y, con ello, la producción de los molinos tubulares, crece en función exponencial de exponente 3,5 aproximadamente, mientras la sección libre lo hace sólo con exponente 2 .

La superficie exterior del molino sobre la cual irradia el calor tampoco aumenta proporcionalmente con la producción, esto es, la disipación de calor por radiación y convección, referida a la producción, empeora con el aumento del diámetro del molino.

Para eliminar, entonces, la suficiente cantidad de calor es necesario inyectar agua al molino. La cantidad de agua a inyectar está limitada por el punto de rocío del aire de escape. Por otra parte, la resistencia en el cemento disminuye con creciente exceso de agua inyectada.

En las instalaciones que tienen electrofiltros para el desempolvado, siempre es necesario el acondicionamiento del aire de escape mediante la inyección de agua al molino.

Por lo tanto, y por los motivos señalados, en los molinos Compound grandes la temperatura del clínker de alimentación debe limitarse, si se quieren evitar defectos en la calidad del cemento ocasionados por temperaturas demasiado elevadas.

\section{Molinos de dos cámaras con separador (Circuito cerrado)}

Las desventajas de los molinos Compound antes enumeradas condujeron al desarrollo de la molienda en circuito cerrado con separador.

El separador hace posible una variación de la finura del cemento, al tiempo que la mantiene constante y evita, con seguridad, el grano grueso. Dentro de ciertos límites es posible la variación de la granulometría entre 2 y 30 micras, que es la porción que más importancia tiene para la resistencia. La economía de la instalación es muy buena.

La figura 13 representa el esquema de una instalación característica. La disposición es la misma que en la molienda de crudo. En la molienda de cemento, la necesidad de frecuentes cambios de la finura adquiere especial importancia, por lo que el separador suele ser del tipo regulable a distancia. El método de regulación más difundido es el de variación de la velocidad del plato dispersor con las paletas de contracorriente. Con ello, se consigue una regulación de la finura en el producto terminado, sin escalones y en un amplio campo. Hay separadores funcionando, que sobre la marcha permiten una variación de la superficie específica entre los $2.600 \mathrm{~cm}^{2} / \mathrm{g}$ del cemento de inferior calidad hasta unos $6.000 \mathrm{~cm}^{2} / \mathrm{g}$ según Blaine. 
En la figura 15 puede verse un separador centrífugo del tipo clásico, que se caracteriza por la circulación interna del aire. La corriente es producida por la turbina en la parte superior. Los gruesos son extraídos del cono interior y los finos del cono de la envolvente.

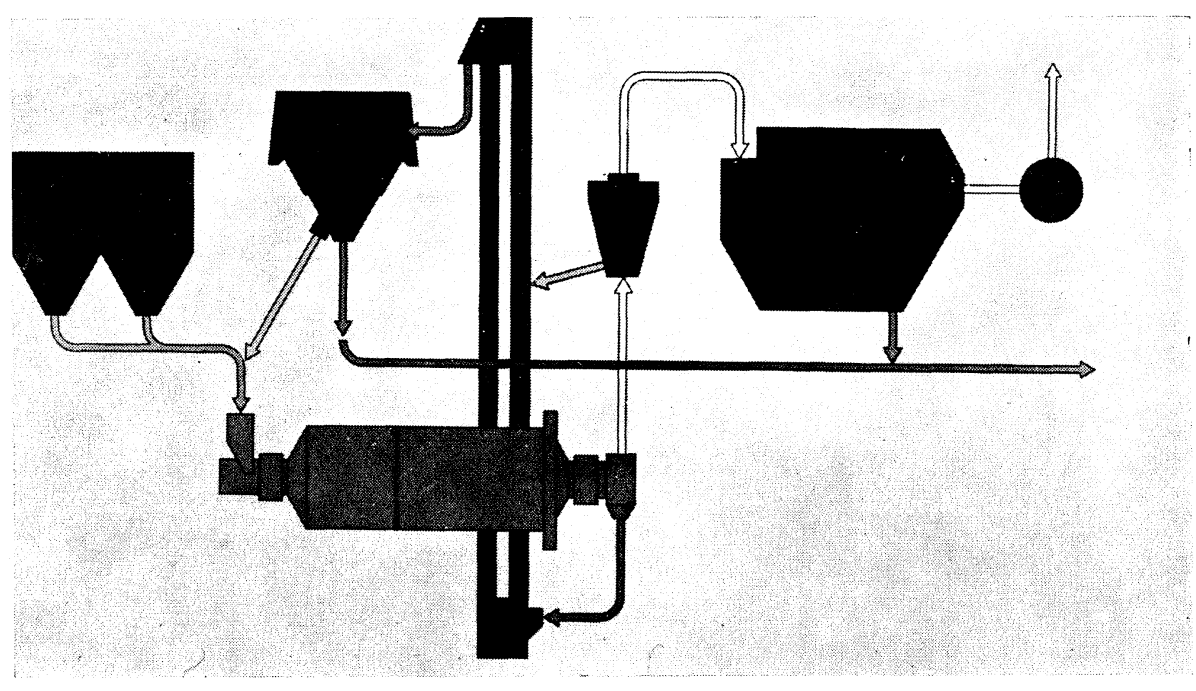

Fig. 13.-Esquema de un molino con turbo-separador.

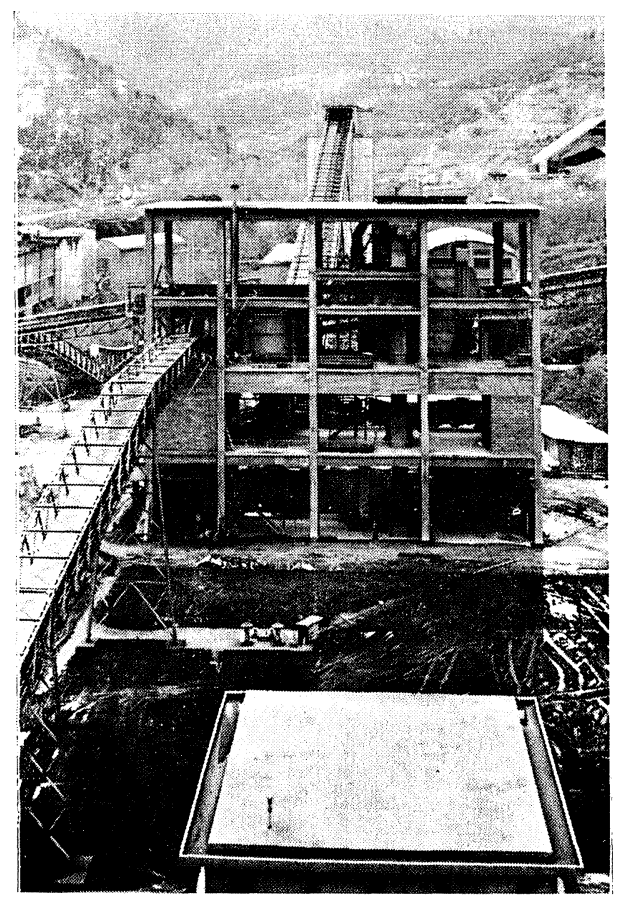

Fig. 14.-Foto de un molino con turbo-separador.

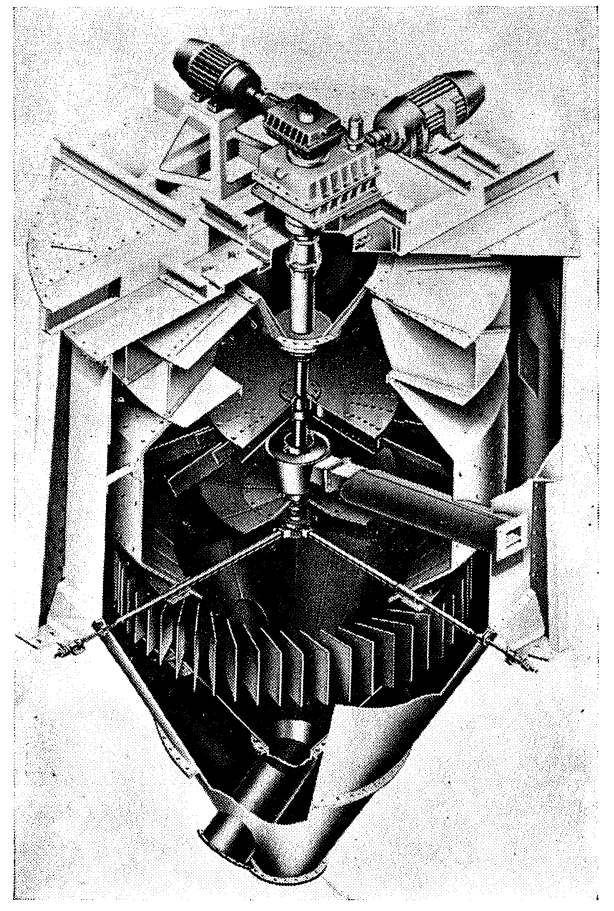

Fig. 15.-Turbo-separador.

Se ha introducido en los últimos años un separador centrífugo, que se distingue por la creación externa de la corriente de aire y la captación de los finos, también externa, en unos ciclones dispuestos circularmente. A pesar de las ventajas que esta disposición ofrece, sobre todo en las unidades de mayor producción, no son suficientes para haber logrado desbancar al separador del tipo clásico. 
El molino suele ser, hoy en día, el de dos cámaras, de las cuales la primera está equipada con un blindaje de placas elevadoras y con una carga de bolas de entre 50 y $100 \mathrm{~mm}$ de diámetro, y la segunda con un blindaje autoclasificador de bolas y una carga de bolas de entre 20 y $50 \mathrm{~mm}$ de diámetro.

La figura 16 es una sección por un molino de dos cámaras. La relación longitud-diámetro de estos molinos se mantiene prácticamente constante hasta en los molinos de gran diámetro. El grado de llenado de bolas oscila, normalmente, entre 29 y $33 \%$ y la velocidad del molino entre el 71 hasta el $76 \%$ de la velocidad crítica.

El molino es accionado, en este caso, por un motor anular. Este motor permite variar la velocidad del molino. Los ensayos realizados con distintas velocidades demuestran, que, si dentro de ciertos límites el rendimiento específico del molino no varía, sí lo hace la potencia absorbida y, con ello, la producción. Es decir, al aumentar las revoluciones, aumenta proporcionalmente la potencia absorbida y la producción, hasta llegar el momento en el que, aunque la potencia siga subiendo, la producción se mantiene estacionaria.

La regulación de la instalación es fácilmente realizable a través de la medición de la carga del elevador, o bien de la cantidad de los gruesos, mediante una báscula o un caudalímetro intercalado en el retorno de gruesos al molino. La alimentación se regula, entonces, de tal forma, que la suma de alimentación y retorno queda constante. Con la ayuda de una escucha eléctrica se vigila el grado de llenado del molino. En caso de sobrecarga se reduce automáticamente la alimentación.

En las figuras 14 y 17 pueden verse sendas fotografías de una instalación de molienda de clínker en circuito cerrado y de un molino de dos cámaras con motor anular, respectivamente.

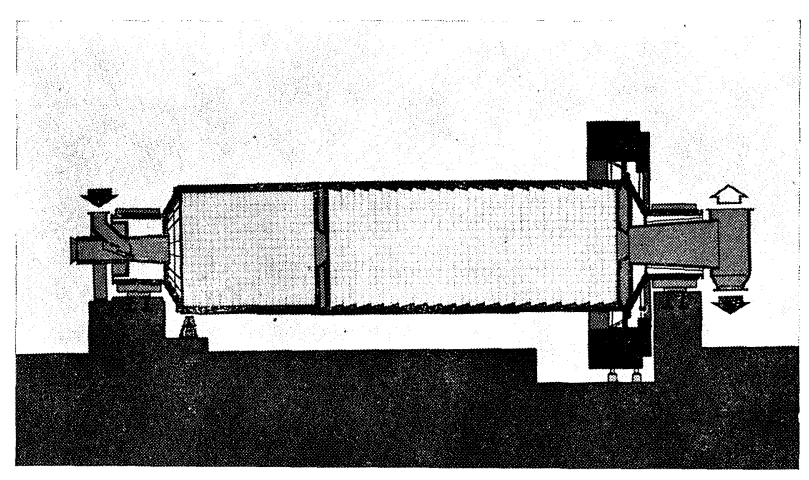

Fig. 16. - Sección de un molino de dos cámaras.

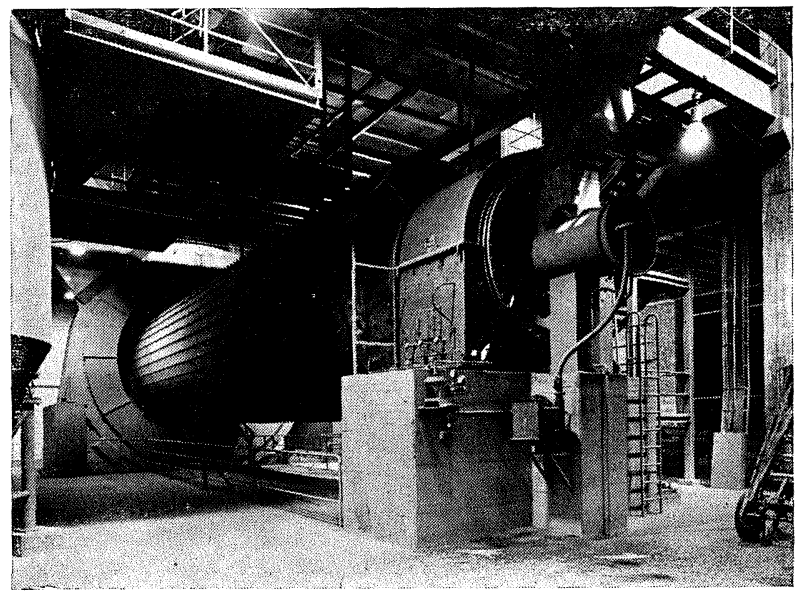

Fig. 17.-Foto de un molino de dos cámaras.

De ensayos comparativos entre molinos de pequeña y gran producción no resultaron diferencias en las calidades del cemento. Solamente en el caso de cambiar de cemento normal a super-cemento, los molinos pequeños se comportan mejor.

Como ya se mencionó en el caso de los molinos Compound, es preciso eliminar una considerable cantidad de calor. Así, en los molinos con separador representa una ventaja el poder admitir velocidades más altas de aire en el recinto de molienda, lo que significa un mayor caudal y una mayor cantidad de calor eliminada. Además, es mayor la superficie de irradiación. Igual que en el caso de los molinos Compound, se inyecta agua al molino para rebajar la temperatura. 
En la tabla 2, y como ejemplo, figura el balance térmico de una instalación de molienda. En este caso, el calor aportado del material y de la molienda es del mismo orden de magnitud. Por motivos de resistencia del cemento se inyecta tan sólo una pequeña cantidad de agua. La mayor parte del calor permanece en el material que sale del molino. El resto es eliminado por el aire y por radiación y convección. En los molinos con separador se nos ofrece, sin embargo, la posibilidad de eliminación del calor del material que se encuentra en el circuito.

En la figura 18 se ve un ejemplo para esta posibilidad.

En el retorno de gruesos está intercalado un enfriador, que consiste en un recipiente con fondo de fluidificación neumática y con haz de tubos de agua para la refrigeración indirecta del material.

\section{T A B L A 2}

Balance térmico de una instalación de molienda de clínker en $\mathrm{kcal} / \mathrm{t}$ cemento producido

\begin{tabular}{|c|c|c|}
\hline \multirow[t]{5}{*}{1.} & Calor aportado & $\mathrm{kcal} / \mathrm{t}$ \\
\hline & 1.1. Accionamiento & 22.200 \\
\hline & 1.2. Material alimentado & 22.000 \\
\hline & 1.3. Aire a la entrada & 400 \\
\hline & & 44.600 \\
\hline \multirow[t]{6}{*}{2 . } & Calor eliminado & \\
\hline & 2.1. Evaponación de agua & 7.500 \\
\hline & 2.2. Material a la salida & 19.500 \\
\hline & 2.3. Aire a la salida & 10.000 \\
\hline & 2.4. Irradiación y convección & 7.600 \\
\hline & & 44.600 \\
\hline
\end{tabular}

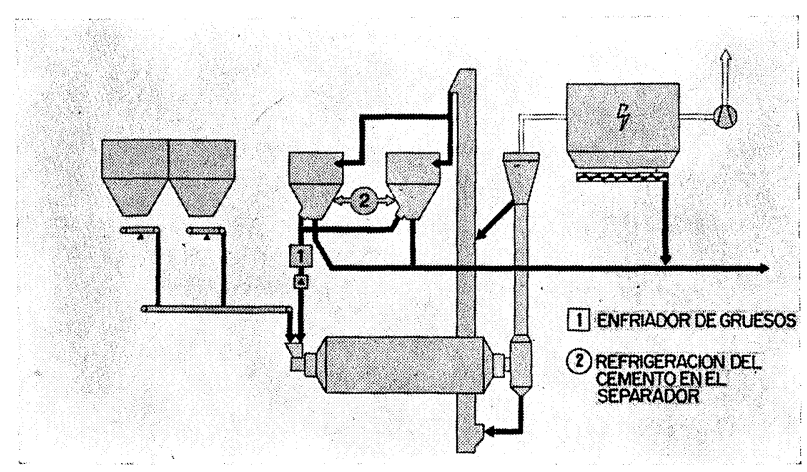

Fig. 18.-Esquema de una instalación de molienda con refrigeración en el circuito.

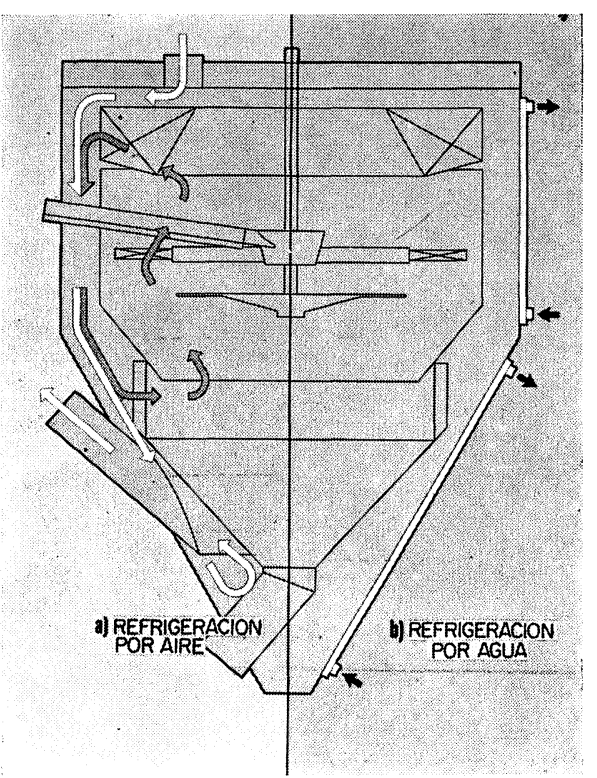

Fig. 19.- Separador con refrigeración. 
El producto terminado se puede enfriar en el separador (fig. 19).

En la parte derecha se puede contemplar la disposición de una segunda envolvente, dejando una cámara por la cual discurre el agua de refrigeración. Puesto que el cemento se desliza en una capa muy delgada por el interior de la carcasa, el intercambio de calor es muy intenso. De esta manera se consigue rebajar la temperatura del cemento hasta en $40^{\circ} \mathrm{C}$.

En la parte izquierda está representada la refrigeración por aire. La reducción de temperatura en este caso es de hasta $20^{\circ} \mathrm{C}$. Tiene la desventaja de que es necesario desempolvar el aire de refrigeración.

La tabla 3 recoge unos datos de una instalación equipada con un enfriador de gruesos y un separador con refrigeración por agua. No se inyecta agua al molino en este caso para evitar deterioro de la resistencia. Esta instalación produce cemento normal y funciona con una carga circulante de 1,75 .

TAB L A 3

Refrigeración en el circuito

\begin{tabular}{ll}
\hline 1. Producción & $157 \mathrm{t} / \mathrm{h}$ \\
2. Aireación del molino & $66.000 \mathrm{~m} / \mathrm{h}$ \\
3. Temperatura del clínker & $140^{\circ} \mathrm{C}$ \\
4. Temperatura del material en circulación & $118^{\circ} \mathrm{C}$ \\
5. Temperatura de los gruesos & $54^{\circ} \mathrm{C}$ \\
6. Temperatura del cemento & $90^{\circ} \mathrm{C}$ \\
7. Cantidad de calor eliminada & \\
7.1. en enfriador de gruesos & $2.225 .000 \mathrm{kcal} / \mathrm{h}$ \\
7.2. en el separador & $1.270 .000 \mathrm{kcal} / \mathrm{h}$ \\
8. Cantidad de agua necesaria & $215 \mathrm{~m}^{3} / \mathrm{h}$ \\
8.1. enfriador de gruesos & $49 \mathrm{~m}^{3} / \mathrm{h}$ \\
\hline
\end{tabular}

La tabla 4 resume los datos generales de algunas instalaciones modernas de molienda de clínker.

TA B L A 4

Instalaciones modernas para la molienda de clínker

\begin{tabular}{|c|c|c|c|c|c|c|c|}
\hline Planta & Dimensiones & Tipo de molino & $\begin{array}{l}\text { Acciona- } \\
\text { miento } \\
k W\end{array}$ & $\begin{array}{c}\text { Tipo de } \\
\text { accionamiento }\end{array}$ & $\begin{array}{l}\text { Finura } \mathrm{cm}^{2} / \mathrm{g} \\
\mathrm{s} / \text { Blaine }\end{array}$ & $\begin{array}{c}\text { Producción } \\
t / h\end{array}$ & Observaciones \\
\hline 1 & $4,8 \times 16,25$ & Compound & 5.600 & Motor anular & 3.200 & 150 & \\
\hline 2 & $4,4 \times 16,0$ & Separador & 4.400 & 2 Piñones (s) & 2.700 & 140 & $\begin{array}{l}\text { Separador refrigerado } \\
\text { por agua }\end{array}$ \\
\hline 3 & $4,6 \times 14,5$ & Separador & 4.200 & 2 Piñones & 3.000 & 130 & $\begin{array}{l}\text { Separador refrigerado } \\
\text { por aire }\end{array}$ \\
\hline 4 & $4,6 \times 14,0$ & Separador & 4.200 & 2 Piñones & 2.800 & 120 & $\begin{array}{l}\text { Secado de esccria en } \\
\text { el molino }\end{array}$ \\
\hline 5 & $4,8 \times 16,5$ & Separador & 5.600 & 2 Piñones (s) & 3.000 & 160 & \\
\hline 6 & $4,8 \times 15.0$ & Separador & 5.000 & Central & 3.000 & 150 & \\
\hline 7 & $5,2 \times 16,5$ & Separador & 6.325 & Motor anular & 3.000 & 250 & \\
\hline
\end{tabular}




\section{DISEÑO CONSTRUCTIVO}

Para el accionamiento de los grandes molinos existen varias soluciones, como ya vimos en la tabla 4.

Los conocidos accionamientos con corona dentada y dos piñones se emplean actualmente hasta potencias de $5.600 \mathrm{~kW}$; con piñones de dientes templados se pueden transmitir incluso potencias aún más altas. En este campo y con los métodos de templado hoy conocidos, podemos esperar un desarrollo interesante. Hasta unos $6.000 \mathrm{~kW}$ de potencia transmitida llegan los accionamientos centrales hoy en día en funcionamiento. Pero también aquí el empleo de dientes templados ofrece la posibilidad de llegar a potencias transmisibles más altas todavía.

Aparte de esto, se ha desarrollado un nuevo tipo de accionamiento sin reductor que se puede emplear hasta potencias extremas: el motor anular ya mencionado.

La figura 20 es una fotografía de un primer plano de un motor sincrono de pequeña velocidad, alimentado con una corriente de baja frecuencia, producida en una instalación de convertidores. El rotor de este motor va directamente fijado a uno de los testeros del molino.

También para las demás partes fue necesario encontrar soluciones nuevas o mejoras en el diseño y materiales. En este sentido, la utilización de las calculadoras electrónicas ha supuesto un gran avance con vistas al correcto dimensionamiento de, por ejemplo, testeros y cilindro de los molinos grandes. Esto, junto con los perfeccionamiento de las técnicas de fabricación y control de calidad, da una aceptable garantía para una alta seguridad de funcionamiento de estas piezas.

También el problema del transporte ha obligado a los constructores a buscar soluciones adecuadas. Los cilindros de los grandes molinos ya se transportan en virolas, que se ensamblan, sueldan y mecanizan en obra.

Son notables, igualmente, los progresos conseguidos en la calidad de los blindajes y de los cuerpos moledores.

Los tabiques de separación entre las cámaras deben resistir a los elevados esfuerzos mecánicos y permitir —en el caso de los molinos secadores- el paso del gas, lo que requiere grandes secciones libres. Además, se debe tener en cuenta los esfuerzos por dilataciones térmicas. En los molinos de gran diámetro los constructores se han visto confrontados con problemas en este sentido, que han tenido que ser superados.

En lo que se refiere a los cojinetes, se han llegado a construir y poner en servicio cojinetes de collar hidrodinámicos de $3,4 \mathrm{~m}$ de diámetro. Pero aparte, se introducen los cojinetes hidrostáticos. Ultimamente, también se ha vuelto a los aros de rodadura, para lo cual han sido desarrollados cojinetes hidrostáticos especiales. Este tipo de soporte admi-

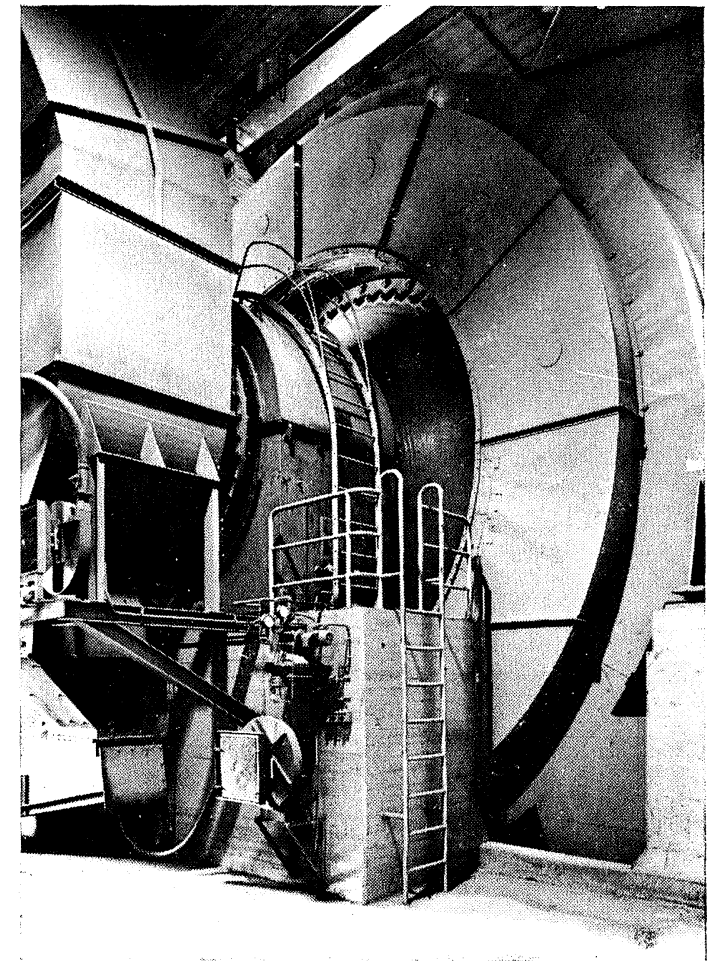

Fig. 20.-Foto de un molino con motor anular (Rohzem II) 
te las más altas cargas y, asimismo, tiene la ventaja de dejar los testeros libres de los esfuerzos mecánicos y admitir mayores secciones libres para el paso de gases.

También los aparatos y máquinas auxiliares necesarias, como transportadores, separadores, filtros, etc., han sido adaptados a las unidades de gran producción, por lo que se puede asegurar que, desde el punto de vista mecánico, no existen problemas insalvables para la construcción de molinos de bolas con potencias absorbidas de hasta $10.000 \mathrm{~kW}$.

\section{PERSPECTIVAS FUTURAS}

El desarrollo en la técnica de la molturación se ha caracterizado en los últimos años por la tendencia hacia unidades cada vez más grandes y, desde el lado mecánico, no existen serios obstáculos para que continúe el aumento de tamaños de los molinos. Tampoco desde el lado de proceso se vislumbra hasta la fecha una limitación en el tamaño. Los molinos grandes no muestran deficiencias en cuanto a la calidad del cemento producido se refiere.

Con respecto al diseño y construcción se ha progresado bastante, tanto en la configuración, como en la ejecución de muchos de los elementos que componen las máquinas. Habrá que prestar ahora atención especial a simplificar y facilitar el mantenimiento de las instalaciones y en aumentar su disponibilidad.

Continúan los esfuerzos en lograr ahorros de energía y mejoras en la calidad, especialmente del cemento. Deben mencionarse aquí, los desarrollos en los campos de los blindajes, los tabiques de separación, los turboseparadores y, también, la investigación de los coadyuvantes de molienda. La introducción del motor anular ha significado, en este sentido, una valiosa posibilidad de estudiar en la práctica la influencia de la velocidad en las condiciones más variadas.

Mediante modelos matemáticos se intenta encontrar, para el molino propiamente dicho y para el circuito, las condiciones óptimas de funcionamiento. Va a ser necesario hacer aplicable a la práctica los resultados que se obtengan.

Por el momento no se vislumbran procedimientos de molienda completamente nuevos que sean aplicables a escala industrial. Los resultados de ensayos con, por ejemplo, los molinos a contrachorro no dejan entrever una conclusión clara sobre las posibilidades de aplicación de este procedimiento. De manera que el desarrollo en la próxima década estará marcado por la mejora de los sistemas de molienda conocidos. 
1. Abierto el Coloquio por el Presidente, Sr. GARCIA MONTON, lo inició el Sr. FERNANDEZ CANOVAS diciendo que los motores síncronos utilizados para el arrastre de molinos y que funcionan a bajas frecuencias logradas mediante convertidores, presentan el problema del arranque. Preguntó cómo se realizaba éste, si como en el caso de un motor asíncrono, o si por medio de otro motor auxiliar.

Contestaron los autores de la ponencia diciendo que en el caso del motor anular no existe problema de arranque, ya que éste puede hacerse desde la velocidad cero, sin escalones, para llegar al sincronismo, mediante conversión de la frecuencia.

2. El Sr. FERNANDEZ CANOVAS planteó otra cuestión en relación con los problemas de los cojinetes de los molinos, al preguntar que qué inconveniente había en utilizar, como en el caso de los hornos, rodillos de apoyo, para aliviar en parte la carga sobre los propios cojinetes.

Los Sres. TIGGSBAUMKER y CANTALAPIEDRA respondieron que el inconveniente estribaba en la velocidad de giro, la cual, siendo baja en los hornos, no crea problemas; pero que al ser alta en los molinos se producirían marcas en los rodillos que acabarían haciendo vibrar a los molinos. Además, el problema se transladaría a los cojinetes de los rodillos. Añadieron que, en lugar de rodillos, se emplean ya apoyos hidrostáticos que, por inyección de aceite a alta presión mantienen a flote el aro de rodadura.

3. El Sr. DANEYKO aclaró después cómo se efectúa el arranque de motores anulares, diciendo que éstos pueden tener un segundo devanado de amortiguación que permite el arranque de forma asíncrona, antes de llegar a la excitación del rotor para producir el sincronismo. Añadió que, en ocasiones, incluso el mismo devanado de excitación puede conectarse o conmutarse de forma que sirva de devanado de amortiguación. De estas formas se logra arrancar a muy baja frecuencia producida por el equipo de conversión, y haciéndolo de forma asíncrona.

4. El Sr. MARTINEZ YNZENGA preguntó después cuál era el fundamento para decir que la inyección de agua en el molino puede afectar a la calidad del cemento.

Los autores respondieron que esa era una cuestión frecuentemente debatida y en la que había opiniones opuestas. Que ellos se basaban en las objecciones que a veces algunos clientes hacen a la inyección de agua, aduciendo deterioro de la calidad del cemento, para decir que en algunas instalaciones parece que se da ese problema.

El Sr. MARTINEZ YNZENGA no consideró contestada su pregunta y dijo que tal vez alguien pudiera dar una explicación a una cuestión de tanto interés y nada clara. $\mathrm{Y}$, añadió que desde un punto de vista práctico parecía evidente que la inyección de agua en el molino no afectaba a la calidad del cemento, y que insistía en que alguien conocedor del tema dijera algo al respecto.

A esto respondió el Sr. TIGGSBAUMKER que el asunto era polémico dentro de la industria cementera, y que no entraba en él ni en la teoría de si la inyección de agua podía afectar o no a la calidad del cemento: que simplemente tenía que basarse en lo 
que dicen al respecto los expertos en la materia, y que si en algún caso concreto algunos de éstos opinan que la calidad del cemento se ve afectada por la inyección de agua, y en consecuencia la rechazan, él tenía que aceptarlo así y que, por consiguiente, en la ponencia presentada se había expuesto la posibilidad de evitar la inyección de agua para rebajar la temperatura del molino. Concluyó diciendo que polemizar más a fondo sobre el tema llevaría demasiado tiempo.

A juicio del Sr. AGUANEL la pregunta quedaba sin contestar, a lo que el Presidente dijo que más bien había que considerar que las respuestas no habían aclarado suficientemente la cuestión, recabando una vez más intervenciones al respecto.

Habló finalmente el Sr. BALAGUER para dar su opinión en el sentido de que una pulverización de agua en el molino, y según la temperatura del clínker, puede dar lugar a la hidratación de las partes superfinas del mismo, lo cual supone indiscutiblemente una merma de resistencia del cemento, ya que dichas partes no se hidratan después de forma activa.

Con esta última intervención y sin considerar del todo dilucidado el asunto, el Presidente, Sr. GARCIA MONTON agradeció las actuaciones de todos los participantes en el Coloquio y dió éste por terminado. 